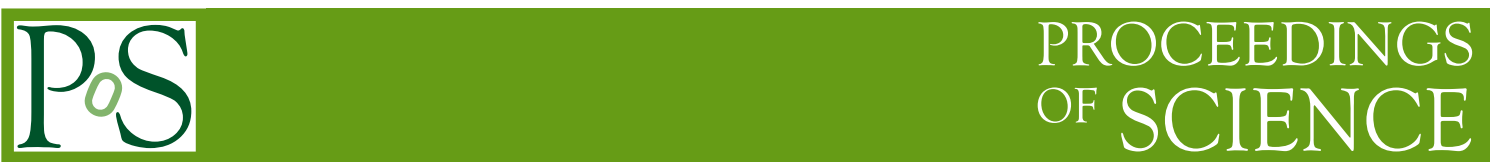

\title{
Geometric effects in lattice QCD thermodynamics
}

\section{Marco Panero*}

Institute for Theoretical Physics, University of Regensburg, 93040 Regensburg, Germany

E-mail: marco.panero@physik . uni-regensburg.de

I present a study of the equation of state in quenched QCD, discussing some systematic effects related to the lattice geometry. In particular, I comment on the modification of the Stefan-Boltzmann law for a gas of free gluons in a finite system, and study the impact it might have on numerical results at high temperatures, for the typical parameters of current lattice simulations. Finally, I apply the results of this study to the analysis of data obtained from simulations of $\operatorname{SU}(N)$ gauge theories with $N>3$ colors, in a temperature range up to $3 T_{c}$, where infrared effects appear to be under control. Preliminary results for various thermodynamic observables for SU(4), SU(5) and $\mathrm{SU}(6)$ gauge theories are found to be close to each other and to those for SU(3), in agreement with other similar studies. This may be relevant for the theoretical description of the QCD plasma.

The XXVI International Symposium on Lattice Field Theory

July 14 - 19, 2008

Williamsburg, Virginia, USA

${ }^{*}$ Speaker. 


\section{Introduction and motivation}

Experiments at temperatures $T$ up to a few times the deconfinement temperature $T_{c}$ reveal a strongly interacting system [1-4], which behaves as a nearly perfect fluid [5]. In this regime, lattice calculations are essentially the only tool to determine the equation of state (EoS) from the first principles of QCD.

In particular, for the gluon sector of the QCD plasma, the lattice results show strong deviations from the Stefan-Boltzmann (SB) limit, with a large deficit in the entropy and pressure [6]. This led to conjecture [7] that the gluon plasma may admit an effective description based on the AdS/CFT correspondence [8]. Together with recent efforts to apply similar gauge/gravity techniques to build a holographic dual of QCD [9], this has triggered interest in lattice studies of $\mathrm{SU}(N)$ Yang-Mills thermodynamics with $N>3$ colors [10-12].

One aspect of the $\mathrm{SU}(N>3)$ simulations is that, in general, they can be carried out using smaller lattices than those needed for $\mathrm{SU}(3)$ - see, e.g. refs. [13, 14]. This should not pose a problem of infrared (IR) effects, because for temperatures of the order of a few times $T_{c}$ finite-volume effects are expected to be exponentially suppressed, due to the existence of screening masses $[15,16]$.

However, it was recently suggested [17] that nontrivial IR corrections affecting a gas of free gluons may still be relevant for the strongly interacting plasma at temperatures $O\left(T_{c}\right)$. For a periodic box of volume $L^{3}$ and timelike size $1 / T$, these corrections depend logarithmically on the aspect ratio of a timelike cross-section $x:=L T$, and vanish for $x \rightarrow \infty$. In this contribution I study numerically the impact of these effects at temperatures up to about $3 T_{c}$.

\section{Finite-volume corrections to the partition function of the free gluon gas}

In the continuum, the partition function $\mathscr{Z}$ for a gas of free $\mathrm{SU}(N)$ gluons in a finite box of spacelike sizes $L \times L \times L$ at temperature $T$ was calculated exactly in ref. [17]:

$$
\frac{\ln \mathscr{Z}}{N^{2}-1}=\frac{\pi^{2}}{45}(L T)^{3}-\ln \sqrt{L T}+O\left(e^{-2 \pi L T}\right) .
$$

The leading finite-volume correction to $\ln \mathscr{Z}$ is a logarithmic function of $L T$. Neglecting terms $O\left(e^{-2 \pi L T}\right)$, the pressure $p$, energy density $\varepsilon$, and free energy density $f$ in a finite volume read:

$$
p=\frac{\varepsilon}{3}=\frac{\pi^{2}}{45} T^{4}\left(N^{2}-1\right)\left[1-\frac{15}{2 \pi^{2}(L T)^{3}}\right], f=-\frac{\pi^{2}}{45} T^{4}\left(N^{2}-1\right)\left[1-\frac{45}{2 \pi^{2}(L T)^{3}} \ln (L T)\right] .
$$

Note that $p$ is no longer equal to $-f$, which is in contrast with the usual assumption underlying the determination of the EoS on the lattice with the integral method [18]. The accuracy of these corrections for the $T \rightarrow \infty$ limit on the lattice is manifest when comparing them with, e.g., the finite-volume corrections to $\varepsilon$ obtained by numerical integration in ref. [19]—see fig. 1 in ref. [17].

One may wonder whether these effects can also play a rôle for a gas of strongly interacting gluons at finite temperature-in particular, at relatively low temperatures $O\left(T_{c}\right)$. As the logarithmic corrections appearing on the r.h.s. of eq. (2.1) stem from the regularization of the divergent contribution to the functional integral coming from constant configurations, and periodic b.c. allow the existence of constant configurations also in the interacting system, it may be that a nontrivial IR 
correction still affects the gluon gas at relatively low temperatures. In particular, in ref. [17] it was pointed out that, for the typical parameters of present lattice simulations, this effect could account for a large fraction of the deviations from the SB limit observed at temperatures of the order of $T_{c}$.

\section{Numerical results}

I run simulations of $\mathrm{SU}(N)$ gauge theories with the standard isotropic Wilson action, using an algorithm that combines heat-bath updates [20] for SU(2) subgroups [21] and full-SU $(N)$ overrelaxation updates [22, 23]; part of the simulations at $T=0$ were run using Chroma [24]. The lattice extent in the timelike direction in lattice units was $N_{\tau}=5$ (and, in some cases, 6); the spacelike volume in lattice units was $N_{s}^{3}$, with $N_{s}$ up to 22, 18, 16 and 16 for SU(3), SU(4), SU(5) and $\mathrm{SU}(6)$, respectively. The $T=0$ simulations were run on $N_{s}^{4}$ lattices.

For SU(3), I set the scale using $r_{0}$ [25], while for the other groups I used the string tension [26, 27]. I determined the EoS using the integral method [18], from measurements of the average plaquette at finely separated $\beta$ values: in particular, $n=180$ intervals of amplitude $\Delta \beta=0.005$ were used for $\mathrm{SU}(3)$. The numerical integration was done with the method described by eq. (A.4) in ref. [28], where it was used to measure the interface tension in the Ising model ${ }^{1}$; the errors of this method are $O\left(n^{-4}\right)$. Cutoff effects on the asymptotic normalization of the EoS were corrected using the $R_{I}\left(N_{\tau}\right)$ factor $[10,31]$.

The IR corrections to the EoS can be calculated analytically only for the free-gluon gas; the result obtained in this limit can be considered as an upper bound for possible nontrivial IR effects in the strongly interacting theory at temperatures of the order of $T_{c}$. To check if in this regime the system is sensitive to logarithmic finite-volume corrections, I calculated the pressure from lattices characterized by different values of $L T$ in two different ways. First, assuming that the data are not affected by nontrivial IR effects. Second, assuming maximal sensitiveness, i.e., assuming the same corrections as for the free-gluon gas. ${ }^{2}$ If the IR corrections affecting the gas of free gluons were still relevant at $T \simeq 2 T_{c}$ or $3 T_{c}$, then the latter method should give better consistency among results obtained from different lattices.

This, however, seems not to be the case: the left panel of fig. 1 shows that the discrepancies between the results obtained using the first method are compatible with statistical fluctuations; this still holds up to $T \simeq 3 T_{c}$ (central panel). On the contrary, the second method overcompensates the differences between the two data sets, driving the curves away from each other (right panel). Similar results also hold for SU(4).

Therefore, within the precision of our data, at temperatures up to $T \simeq 3 T_{c}$ we do not observe evidence for the logarithmic IR corrections that affect the free-gluon gas. Yet, as these corrections should eventually show up in the perturbative regime at high enough temperatures, it would be interesting to study at what $T$ values they can be seen on the lattice. Since the logarithmic corrections are nonnegligible only for $L T=O(1)$, this problem could be studied by running lattice simulations

\footnotetext{
${ }^{1}$ See refs. $[29,30]$ and references therein for further details.

${ }^{2}$ Since the quantity that is measured on the lattice is $-f$, rather than $p$, the latter method requires that the data be shifted (to compensate for $p+f \neq 0$ ) and rescaled (to account for the different high-temperature limit).
} 

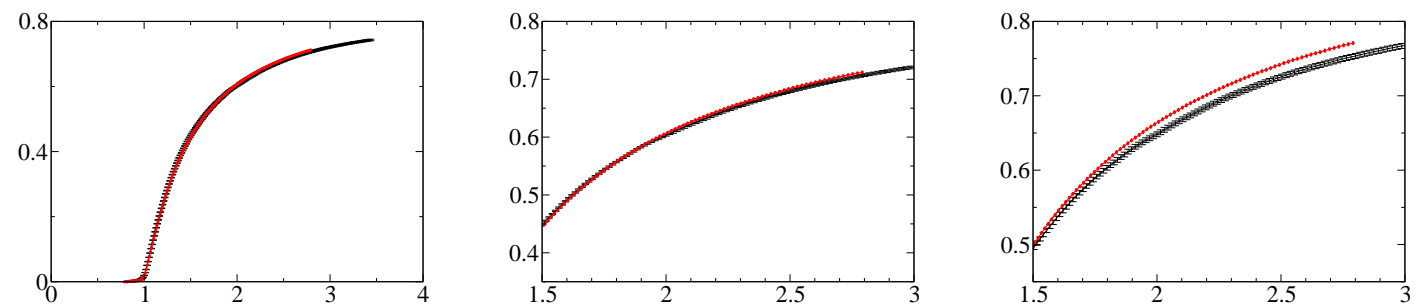

Figure 1: Left panel: The EoS for $\mathrm{SU}(3)$, evaluated neglecting possible logarithmic corrections to the partition function, for lattices of size $20^{3} \times 5$ (black crosses) and $18^{3} \times 5$ (red circles). The plot shows the results for $p / T^{4}$ (normalized to the SB limit) vs. $T / T_{c}$. Central panel: High-temperature zoom of the previous plot. Right panel: The EoS evaluated assuming maximum sensitiveness to IR effects (i.e. compensating for the logarithmic effects that affect a free-gluon gas), according to eq. (2.2).
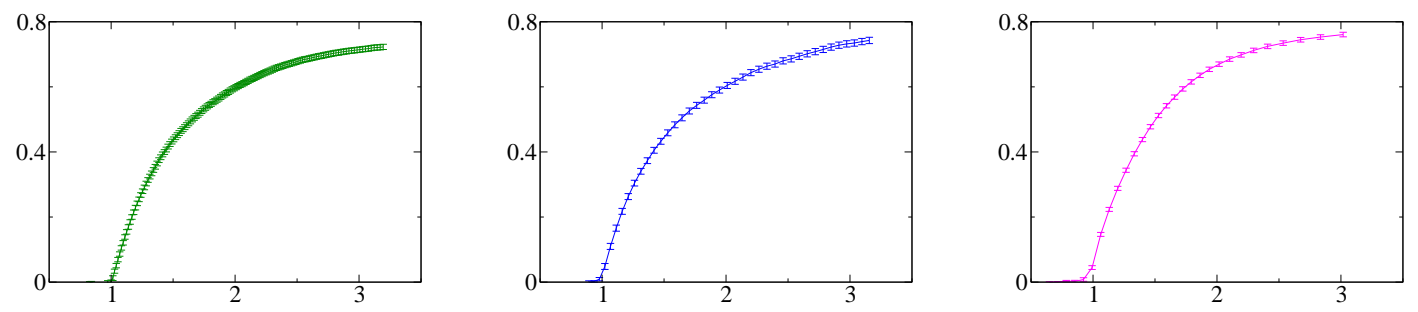

Figure 2: The rescaled pressure $p / T^{4}$ (normalized to the SB limit) from simulations on a $16^{3} \times 5$ lattice, $v s$. $T / T_{c}$, for $\mathrm{SU}(4)$ (left panel), $\mathrm{SU}(5)$ (central panel) and $\mathrm{SU}(6)$ (right panel).

at large $\beta$ values and shrinking physical volume. ${ }^{3}$ Although such a limit has its own reasons of theoretical interest [34,35], in the present work I restricted my analysis to temperatures $O\left(T_{c}\right)$, and to sufficiently large physical volumes.

Having found no evidence for logarithmic IR corrections in the $T<3 T_{c}$ regime, I proceeded to the evaluation of thermodynamic observables in $\mathrm{SU}(N)$. Figs. 2-5 show preliminary results for equilibrium thermodynamics observables (pressure, trace of the stress tensor $\Delta=\varepsilon-3 p$, energy density and entropy density $s$ ), for $3 \leq N \leq 6$, normalized to their SB limits. In agreement with the conclusions of ref. [10], the results from various gauge groups are essentially compatible with each other (with small quantitative corrections), and clearly different from the SB limit. Further results, including data for $\mathrm{SU}(7)$ and $\mathrm{SU}(8)$, will be presented elsewhere [12].

\footnotetext{
${ }^{3}$ The SU(3) EoS at very high temperatures was studied in ref. [32], where the results shown in fig. 5 were obtained from simulations with $N_{\tau}=8$ and $N_{s}^{3}=24^{3}$ or $N_{s}^{3}=32^{3}$ [33]. The maximal IR correction would then shift the asymptotic value down to about $97 \%$ of the SB limit, which indeed looks compatible with their result at the highest temperature $T=3 \times 10^{7} T_{c}$ (although the data are also compatible with the conventional SB limit). Note however, that this comparison is only approximate, because in the numerical method of ref. [32] the geometric parameter $x$ is not held fixed, and furthermore the pressure at high temperatures is obtained using the integral method, starting from low temperatures. This is based on the $p=-f$ equality, which—as we have seen—is reliable in the low- $T$ regime, but would also start being affected by IR corrections when $T$ is increased.
} 

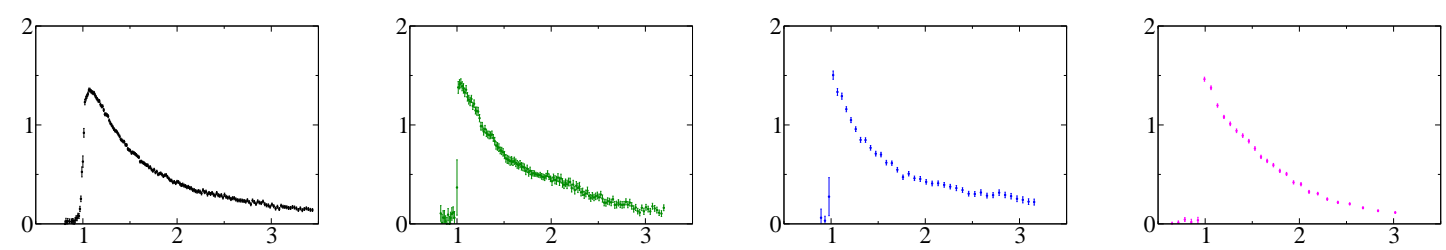

Figure 3: Left to right: $\Delta / T^{4}$, normalized to $\frac{\pi^{2}}{45}\left(N^{2}-1\right), v s . T / T_{c}$, for $\mathrm{SU}(3), \mathrm{SU}(4), \mathrm{SU}(5)$ and $\mathrm{SU}(6)$.
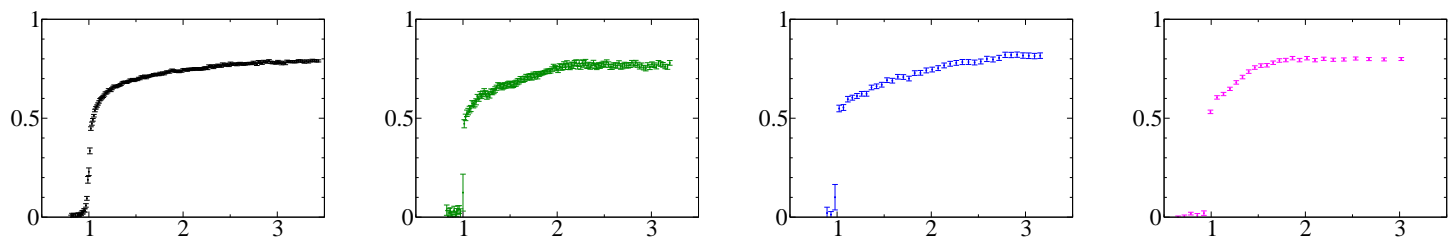

Figure 4: Left to right: The rescaled energy density $\varepsilon / T^{4}$ (normalized to its SB limit) $v s . T / T_{c}$, for $\mathrm{SU}(3)$, $\mathrm{SU}(4), \mathrm{SU}(5)$ and $\mathrm{SU}(6)$.
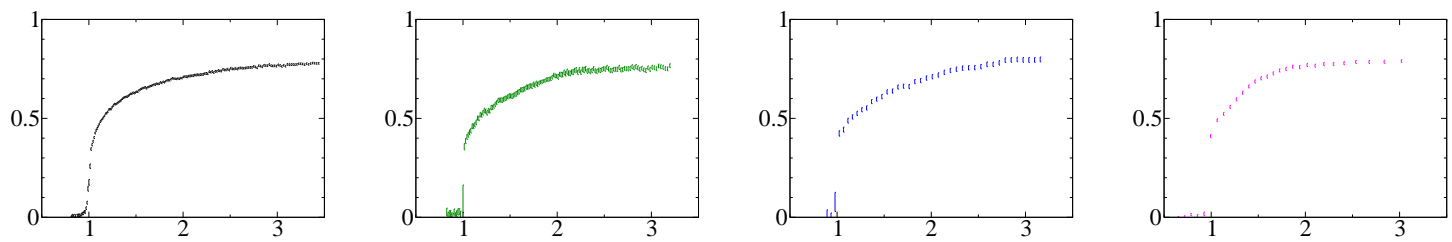

Figure 5: Same as in fig. 4, but for the rescaled entropy density $s / T^{3}$.

\section{Summary and outlook}

The nontrivial IR corrections affecting a gas of free gluons in a finite box were derived in ref. [17], where it was also suggested that they may still play a rôle at temperatures of the same order of magnitude as $T_{c}$. To the level of precision achieved, my numerical results in a (phenomenologically relevant) temperature range up to about $3 T_{c}$ did not reveal any nontrivial finite-volume effects. However, the latter might probably be observed in numerical simulations at very high temperatures and small volumes [32]. Next I also measured bulk thermodynamic quantities in $\mathrm{SU}(N)$ gauge theories with $3 \leq N \leq 6$ colors, at temperatures up to $3 T_{c}$. In agreement with ref. [10], the results for different $\mathrm{SU}(N)$ groups share similar features, and the strong deviations from the $\mathrm{SB}$ limit observed in $\mathrm{SU}(3)$ survive the large- $N$ limit. This leans support to the hypothesis that QCD may admit a nearly conformal effective description at temperatures of a few times $T_{c}$, where the theory is still strongly interacting [36].

\section{Acknowledgments}

I acknowledge support from the Alexander von Humboldt Foundation, and thank G. S. Bali, Ph. de Forcrand, F. Gliozzi, S. Gupta, B. Lucini, K. K. Szabó and G. D. Torrieri for discussions. The University of Regensburg participates in the SFB/TR 55 "Hadronenphysik mit Gitter-QCD". 


\section{References}

[1] K. Adcox et al. [PHENIX Collaboration], Formation of dense partonic matter in relativistic nucleus nucleus collisions at RHIC: Experimental evaluation by the PHENIX collaboration, Nucl. Phys. A 757, 184 (2005) [arXiv:nucl-ex/0410003].

[2] I. Arsene et al. [BRAHMS Collaboration], Quark gluon plasma and color glass condensate at RHIC? The perspective from the BRAHMS experiment, Nucl. Phys. A 757 (2005) 1 [arXiv:nucl-ex/0410020].

[3] B. B. Back et al., The PHOBOS perspective on discoveries at RHIC, Nucl. Phys. A 757, 28 (2005) [arXiv:nucl-ex/0410022].

[4] J. Adams et al. [STAR Collaboration], Experimental and theoretical challenges in the search for the quark gluon plasma: The STAR collaboration's critical assessment of the evidence from RHIC collisions, Nucl. Phys. A 757, 102 (2005) [arXiv:nucl-ex/0501009].

[5] P. F. Kolb and U. W. Heinz, Hydrodynamic description of ultrarelativistic heavy-ion collisions, arXiv:nucl-th/0305084.

[6] G. Boyd, J. Engels, F. Karsch, E. Laermann, C. Legeland, M. Lütgemeier and B. Petersson, Thermodynamics of SU(3) Lattice Gauge Theory, Nucl. Phys. B 469 (1996) 419 [arXiv:hep-lat/9602007].

[7] S. S. Gubser, I. R. Klebanov and A. A. Tseytlin, Coupling constant dependence in the thermodynamics of $N=4$ supersymmetric Yang-Mills theory, Nucl. Phys. B 534 (1998) 202 [arXiv: hep-th/9805156].

[8] J. M. Maldacena, The large N limit of superconformal field theories and supergravity, Adv. Theor. Math. Phys. 2 (1998) 231 [Int. J. Theor. Phys. 38 (1999) 1113] [arXiv: hep-th/9711200].

[9] J. Erlich, E. Katz, D. T. Son and M. A. Stephanov, QCD and a holographic model of hadrons, Phys. Rev. Lett. 95 (2005) 261602 [arXiv: hep-ph/0501128].

[10] B. Bringoltz and M. Teper, The pressure of the $S U(N)$ lattice gauge theory at large-N, Phys. Lett. B 628 (2005) 113 [arXiv: hep-lat/0506034].

[11] S. Datta and S. Gupta, in progress.

[12] M. Panero, in progress.

[13] B. Lucini, M. Teper and U. Wenger, The high temperature phase transition in $S U(N)$ gauge theories, JHEP 0401 (2004) 061 [arXiv: hep-lat/0307017] .

[14] B. Lucini, M. Teper and U. Wenger, Properties of the deconfining phase transition in SU(N) gauge theories, JHEP 0502 (2005) 033 [arXiv: hep-lat/0502003] .

[15] C. E. DeTar, A Conjecture Concerning The Modes Of Excitation Of The Quark-Gluon Plasma, Phys. Rev. D 32 (1985) 276.

[16] J. P. Blaizot and E. Iancu, The quark-gluon plasma: Collective dynamics and hard thermal loops, Phys. Rept. 359 (2002) 355 [arXiv: hep-ph/0101103].

[17] F. Gliozzi, The Stefan-Boltzmann law in a small box and the pressure deficit in hot SU(N) lattice gauge theory, J. Phys. A 40 (2007) F375 [arXiv: hep-lat/0701020].

[18] J. Engels, J. Fingberg, F. Karsch, D. Miller and M. Weber, Nonperturbative thermodynamics of $S U(N)$ gauge theories, Phys. Lett. B 252 (1990) 625. 
[19] B. Beinlich, F. Karsch and E. Laermann, Improved Actions for QCD Thermodynamics on the Lattice, Nucl. Phys. B 462, 415 (1996) [arXiv: hep-lat/9510031].

[20] A. D. Kennedy and B. J. Pendleton, Improved Heat Bath Method For Monte Carlo Calculations In Lattice Gauge Theories, Phys. Lett. B 156 (1985) 393.

[21] N. Cabibbo and E. Marinari, A New Method For Updating SU(N) Matrices In Computer Simulations Of Gauge Theories, Phys. Lett. B 119 (1982) 387.

[22] J. Kiskis, R. Narayanan and H. Neuberger, Does the crossover from perturbative to nonperturbative physics in QCD become a phase transition at infinite N?, Phys. Lett. B 574 (2003) 65 [arXiv:hep-lat/0308033].

[23] Ph. de Forcrand and O. Jahn, Monte Carlo overrelaxation for $S U(N)$ gauge theories, arXiv:hep-lat/0503041.

[24] R. G. Edwards and B. Joó [SciDAC Collaboration and LHPC Collaboration and UKQCD Collaboration], The Chroma software system for lattice QCD, Nucl. Phys. Proc. Suppl. 140 (2005) 832 [arXiv:hep-lat/0409003].

[25] S. Necco and R. Sommer, The $N(f)=0$ heavy quark potential from short to intermediate distances, Nucl. Phys. B 622 (2002) 328 [arXiv: hep-lat/0108008].

[26] B. Lucini, M. Teper and U. Wenger, Glueballs and k-strings in $S U(N)$ gauge theories: Calculations with improved operators, JHEP 0406 (2004) 012 [arXiv: hep-lat/ 0404008 ] .

[27] B. Lucini and M. Teper, The $k=2$ string tension in four dimensional $S U(N)$ gauge theories, Phys. Lett. B 501 (2001) 128 [arXiv: hep-lat/0012025].

[28] M. Caselle, M. Hasenbusch and M. Panero, The interface free energy: Comparison of accurate Monte Carlo results for the 3D Ising model with effective interface models, JHEP 0709 (2007) 117 [arXiv:0707.0055 [hep-lat]].

[29] M. Caselle, M. Hasenbusch and M. Panero, String effects in the 3d gauge Ising model, JHEP 0301 (2003) 057 [arXiv: hep-lat/0211012].

[30] M. Caselle, M. Hasenbusch and M. Panero, On the effective string spectrum of the tridimensional Z(2) gauge model, JHEP 0601 (2006) 076 [arXiv : hep-lat/0510107].

[31] J. Engels, F. Karsch and T. Scheideler, Determination of anisotropy coefficients for SU(3) gauge actions from the integral and matching methods, Nucl. Phys. B 564 (2000) 303 [arXiv:hep-lat/9905002].

[32] G. Endrődi, Z. Fodor, S. D. Katz and K. K. Szabó, The equation of state at high temperatures from lattice QCD, PoS (LAT2007) 228 (2007) [arXiv:0710.4197 [hep-lat] ] .

[33] K. K. Szabó, private communication.

[34] F. Bruckmann, S. Keppeler, M. Panero and T. Wettig, Polyakov loops and spectral properties of the staggered Dirac operator, arXiv:0804.3929 [hep-lat] .

[35] F. Bruckmann, S. Keppeler, M. Panero and T. Wettig, Polyakov loops and SU(2) staggered Dirac spectra, PoS (LAT2007) 274 (2007) [arXiv:0802.0662 [hep-lat] ].

[36] R. V. Gavai, S. Gupta and S. Mukherjee, The speed of sound and specific heat in the QCD plasma: Hydrodynamics, fluctuations and conformal symmetry, Phys. Rev. D 71 (2005) 074013 [arXiv:hep-lat/0412036]. 